\title{
Improved optical fibre sensors using hollow glass spheres with a high-performance CCD spectrometer interrogator
}

\author{
J. P. Dakin ${ }^{1}$, W. Ecke ${ }^{2}$, M. Reuter ${ }^{2}$, and K. Schroeder ${ }^{2}$ \\ ${ }^{1}$ Optoelectronics Research Centre, Univ of Southampton, UK, SO17 1BJ * \\ ${ }^{2}$ Institute for Photonic Technology IPHT, 07745 Jena, Germany **
}

\begin{abstract}
Hollow glass micro-spheres, first used to make fibre optic sensors for high hydrostatic pressure, have been interrogated using a high-precision CCD spectrometer, to give far better precision than earlier. It is found that these simple, low-cost micro-sensors have excellent sensitivity to both static and dynamic pressure, and have the advantage of being hermetically sealed. Many other application areas are foreseen for these low-cost sensors.
\end{abstract}

\section{INTRODUCTION}

Hollow glass micro-spheres are routinely manufactured in large quantities for many industrial applications. The most common use is to incorporate them in polymers to reduce their density (e.g. for marine flotation aids or lightweight vehicles), whilst retaining good mechanical strength. More exotic applications have been also perceived, such as using hydrogen-filled glass micro-bubbles for fusion-energy by laser-induced, high-temperature implosion, and this latter use has probably indicated a more recently perceived large-scale application for safer storage of pressurised hydrogen gas fuel in vehicles.

The only previously reported use of such micro-spheres for fibre sensors was by Xu and Dakin [1], who bonded a single hollow sphere on the distal end of a single-mode optical fibre, to form a compact and low-cost sensor head.

The light originating from a broadband LED source and leaving the fibre end is reflected back into the fibre, now showing spectral changes due to interference fringes from the two Fresnel reflections from opposite sides of the glass sphere (sensor photograph in Fig. 1). The sphere effectively forms a Fizeau interferometer, or what could also be viewed as a low-finesse Fabry Perot cavity. No additional optics is required, as the effects of diffractive beam broadening are very small for the first $150 \mu \mathrm{m}$ or so after emerging from a single-mode fibre, and any slight beam broadening due to diffraction is also partially corrected by the concave far-surface of the sphere.

Fig. 1 Photograph of hollow sphere sensor (right, Potters Q-Cel ${ }^{\circledR}$, type 7019) attached to the end-face of a single-mode optical fibre (left) of diameter $125 \mu \mathrm{m}$.

The glass bubble sensor was first reported some time before the use of fibre grating sensors became commonplace and, at that time, the sensor could only be interrogated using a simple laboratory spectrum analyser. It was
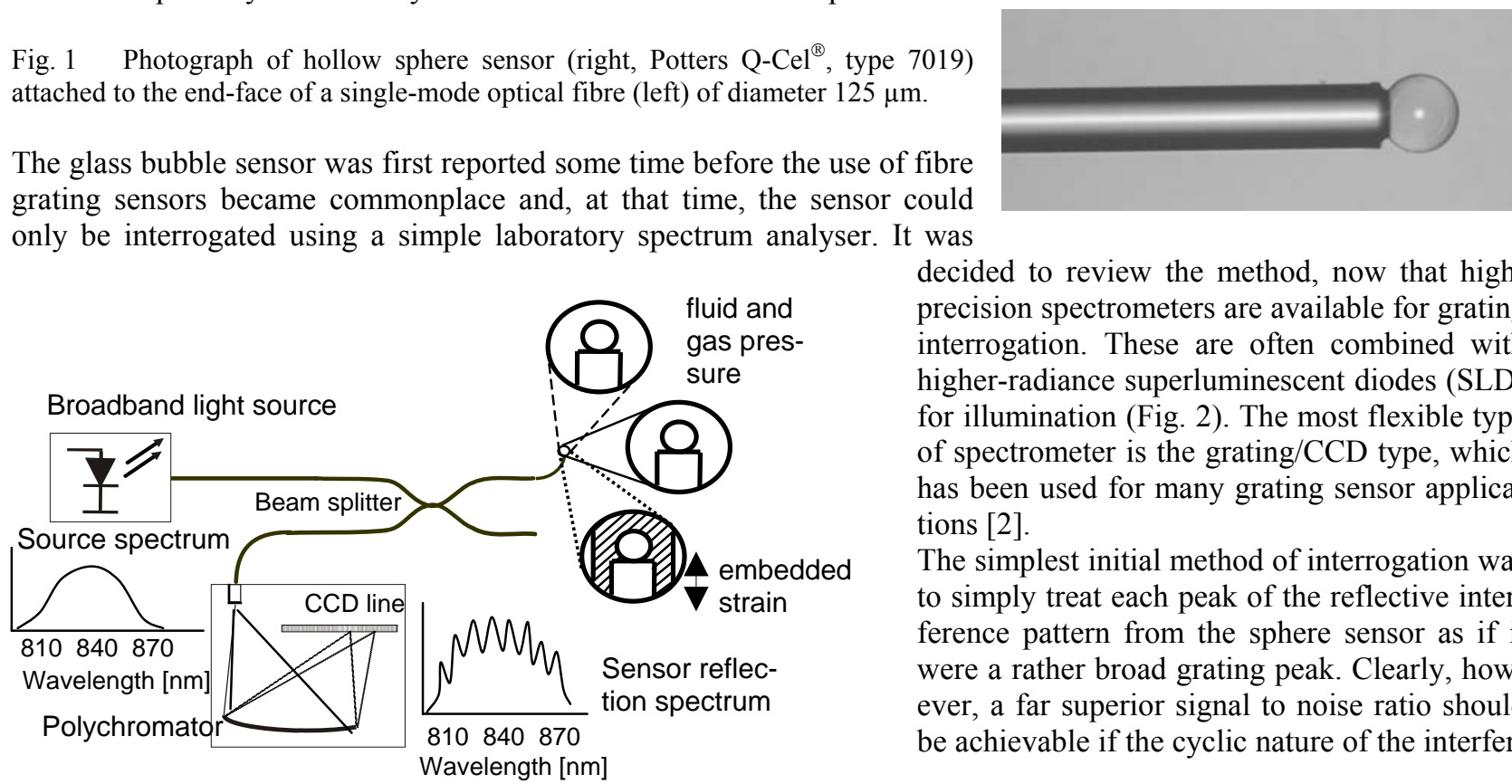

decided to review the method, now that highprecision spectrometers are available for grating interrogation. These are often combined with higher-radiance superluminescent diodes (SLD) for illumination (Fig. 2). The most flexible type of spectrometer is the grating/CCD type, which has been used for many grating sensor applications [2].

The simplest initial method of interrogation was to simply treat each peak of the reflective interference pattern from the sphere sensor as if it were a rather broad grating peak. Clearly, however, a far superior signal to noise ratio should be achievable if the cyclic nature of the interfer-

Fig. 2 Scheme of polychromator based fibre sensor interrogation unit.

\footnotetext{
*jpd@orc.soton.ac.uk_ ${ }^{* *}$ wolfgang.ecke@ipht-jena.de
} 
ence fringes is taken full advantage of, either by using correlation with sine and/or cosine reference waveforms having appropriate phase and spectral spacing, or by numerical averaging of data from all the peaks.

\section{THEORY}

The observed interference occurs between beams reflected from the two inner surfaces of the hollow glass sphere. If the illumination were to be flat and broadband, and assuming the refractive index of the gas in the glass bubble is $\sim 1$, the intensity, $I_{d}$, of the back reflected light is therefore given by

$$
I_{d}=\mathrm{k} \cdot\left[A_{1}{ }^{2}+A_{2}{ }^{2}+2 \cdot A_{1} \cdot A_{2} \cdot \cos (4 \pi \cdot d / \lambda)\right]
$$

where $\mathrm{k}$ is a constant, $\mathrm{A}_{1}$ and $\mathrm{A}_{2}$ represent the electric field amplitudes of the interfering waves that are coupled back into the fibre, $\lambda$ is the wavelength of the source and $d$ is the cavity spacing, which we assume is approximately equal to the inner diameter of the sphere. The free spectral range (FSR) of the cavity is given by

$$
\mathrm{FSR}=\mathrm{c} / 2 \cdot \mathrm{d}
$$

again assuming the refractive index of the gas in the glass bubble is $\sim 1$. Reflectance maxima occur when

$$
\lambda=2 \cdot \mathrm{d} / \mathrm{m}
$$

where $m$ is an integer.

By differentiating equation (3) and substituting for $\mathrm{m}$, the change in wavelength, $\Delta \lambda$, resulting from a small pressure change, $\Delta \mathrm{P}$, is given by

$$
\Delta \lambda=\lambda / \mathrm{d}(\Delta \mathrm{P} \cdot \delta \mathrm{d} / \delta \mathrm{P})
$$

Where $\delta \mathrm{d} / \delta \mathrm{P}$ is the incremental change, $\delta \mathrm{d}$, in internal diameter in response to a small pressure change, $\delta \mathrm{P}$. Generally, the change in diameter, $\delta \mathrm{d}$ will be small, as even very thin-walled spheres can withstand very high pressures. If we assume the pressure on the sphere is isotropic, then the induced diameter change $\Delta \mathrm{d}$, in response to an excess pressure $\mathrm{P}$ is given in [2]

$$
\Delta \mathrm{d}=\mathrm{P} \cdot \mathrm{d}^{2} \cdot(1-\mathrm{v}) / 4 \cdot \mathrm{Y} \cdot \mathrm{t}
$$

where $t$ is the wall thickness and $\mathrm{Y}$ and $v$ are, respectively, the Young's modulus and Poisson ratio of the glass material. The pressure-induced fringe shift, $\Delta \lambda$, is therefore given by

$$
\Delta \lambda=\Delta \mathrm{P} \cdot \lambda \cdot \mathrm{d}(1-v) / 4 \cdot \mathrm{Y} \cdot \mathrm{t}
$$

The sensitivity to pressure is therefore better for larger spheres, provided the wall thickness is the same.

In practice, the reflected spectrum will be modulated by the incident spectrum from the light source, to give an actual intensity spectrum of

$$
I_{d}=k \cdot\left[A_{1}{ }^{2}+A_{2}{ }^{2}+2 \cdot A_{1} \cdot A_{2} \cdot \cos (4 \pi \cdot d / \lambda)\right] \cdot f(\lambda)
$$

where $f(\lambda)$ is the intensity spectrum of the light source. This will give a source-related envelope to the raised-sine waveform predicted from equation 1 and will result in small source-spectrum-related variations in the derived equations above. Clearly, the broader and flatter the source spectrum, $f(\lambda)$, the smaller any corrections will be.

The expected cross-talk to a temperature change, assuming unrestricted thermal expansion of a free-standing sphere, is given by

$$
\Delta \lambda=\Delta \mathrm{T} \cdot(\delta \mathrm{d} / \delta \mathrm{T}) \cdot \lambda / \mathrm{d}
$$

Clearly this will be affected if any glues or cements are used to attach the sphere to the fibre.

\section{EXPERIMENTS}

We have formed new sensors, again using the originally-reported method, with a hollow glass sphere bonded to the distal end of a single-mode fibre. In order to obtain good interference fringe contrast, it is necessary to position the sphere at the end of the fibre, in an on-axis position, before setting the epoxy with UV light. No interference fringes are seen if the centre of the sphere is even a few microns off-axis, as the light beam leaving the fibre core is then effectively refracted off axis, and at least one of the two reflected beams is reflected back to the fibre end at an unsuitable position (or angle) to couple to a guided mode. Once interference fringes are clearly seen in the reflective spectrum (see Fig. 3) the UV cement is set, and the sensor head is enclosed in a protective micro-tube. It is then ready for initial experiments to measure the variation of fringe wavelengths with pressure and temperature. We are able to decide whether to immerse the outer surface or not. Clearly, with the outer surface unmatched, there will be two reflections from closelyspaced interfaces (a few microns, dependent on sphere wall thickness). These may either reinforce or partially cancel, 
depending on their relative phase, making the equations somewhat more complex, particularly as the wall thickness is generally an unknown variable. Even for air pressure measurements, we can cover the glass sphere with oil or soft
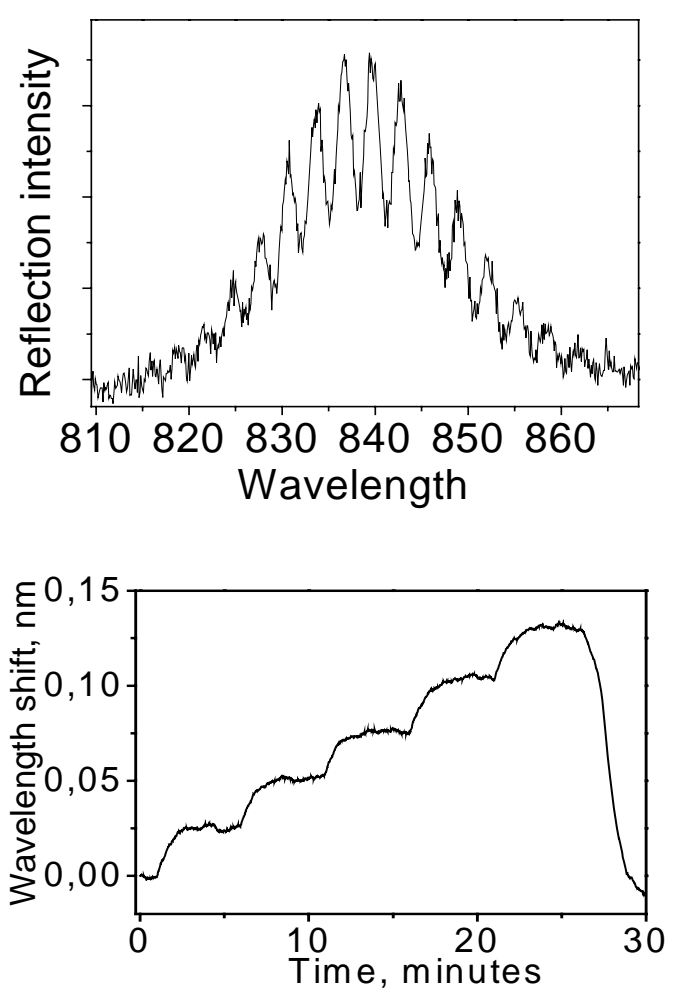

grease to provide index matching.

The reflected spectrum of a typical fibre-remoted sphere sensor in air, when illuminated through the fibre using a SLD light source, is shown in Fig. 3. This clearly shows the regular spacing of the fringes (periodically, indeed, on a $1 / \lambda$ scale) and also the modulation envelope from the source spectrum, as predicted by equation (7) above.

Fig. 3 Reflected spectrum of a typical fibre-remoted sphere sensor (100 $\mu \mathrm{m}$ inner diameter) in air, when illuminated with SLD source.

\section{Use of a sphere as a gas pressure sensor}

We have now used such a hollow sphere as an air pressure sensor, initially over a range of 0 to 0.5 Bar over-pressure (i.e. above atmospheric), increasing it in approximately equal 0.1 Bar steps, using a manual valve and simple Bourdon pressure gauge (Fig. 4).

Fig 4 Wavelength shift on a sphere sensor in air, over a range of 0 to 0.5 Bar over-pressure, in equal 0.1 Bar steps. This gives a noise-limited pressure resolution of order 0.01 Bar at lower over-pressures.

These results were measured using an IPHT-constructed [4] CCD polychromator, with polynomial curve-fitting software, originally designed for grating interrogation to locate the peaks. The pipe fitting in which the sensor was mounted was kept at constant temperature in a water bath, to avoid effects due to adiabatic thermal changes, as increasing the air pressure transiently raises the temperature of the air. The curves all show a similar increase with pressure.

It can be seen that the system noise-limited resolution is extremely small ( $\sim 0.01$ Bar) at the starting 1Bar (zero over-

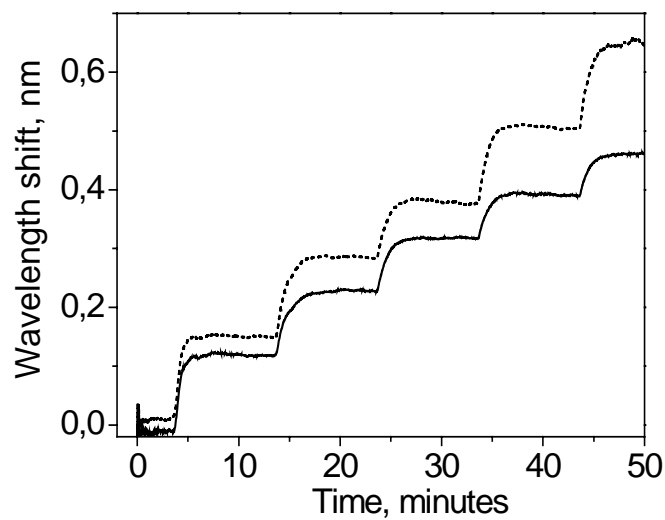

pressure) but increases as pressure increases, a behaviour we believe is due to the minor manual adjustments which had to be made to keep the air in the pressure vessel set close to the desired pressure. Because of the greater precision of the current interrogation system, and because a very thin-walled glass sphere (Potters Q-Cel ${ }^{\circledR}$, type 7019) was used, this resolution is a huge improvement over earlier work, where only very high pressure changes ( $\sim 1$ Bar) could be usefully resolved.

We then repeated the measurement over a wider over-pressure range of 0 to $2.5 \mathrm{Bar}$, in steps of $0.5 \mathrm{Bar}$ (Fig. 5).

Fig 5 Measured wavelength shifts of two different sensors (different wall thickness), as pressure was changed from 0 to 2.5 Bar in 0.5 Bar steps.

\section{Use of a sphere as an embedded strain sensor}

The sphere sensor also has potential as an embedded strain sensor. In order to protect the fibre-sphere sensor head for embedding (direct embedding would put too much strain on the fragile thin-walled sphere) there are several possibilities. The easiest two possibilities are (a) to embed the sphere in a protective tube, which will offer mechanical protection against lateral pressure and (b) to embed the sphere and fibre end in a small droplet of polymer resin, of similar type to that which may be used (with fibre reinforcement) for composite construction.

We have protected such a sensor in a polymer-droplet and then embedded it in a test coupon of glass-fibre-reinforced plastic, using, to ensure compatibility, the same bonding resin as used for the droplet. 
In Fig. 6, a photograph of this realised option (b) is given, as seen through the polymer droplet. A sphere of $134 \mu \mathrm{m}$ outer diameter (left-hand side in Fig. 6) was first bonded on the end of a $125 \mu \mathrm{m}$ diameter fibre (right), then embedded in the polymer droplet, then embedded it in the test coupon of glass-fibre-reinforced polymer. This $15 \times 15 \times 280 \mathrm{~mm}^{3}$ cuboid test coupon was made from polymer resin, reinforced with on-axis glass fibre strands, and was fitted with bonded-in end bolts to make an electrically-insulated device. The excellent isolation of the insulating polymer points to a possible application as a load cell for supporting high-voltage wires.

The measurements of wavelength, versus tensile force on the coupon (Fig. 7) show great improvement of reflective spectra read-out by applying a sinusoidal filter algorithm.
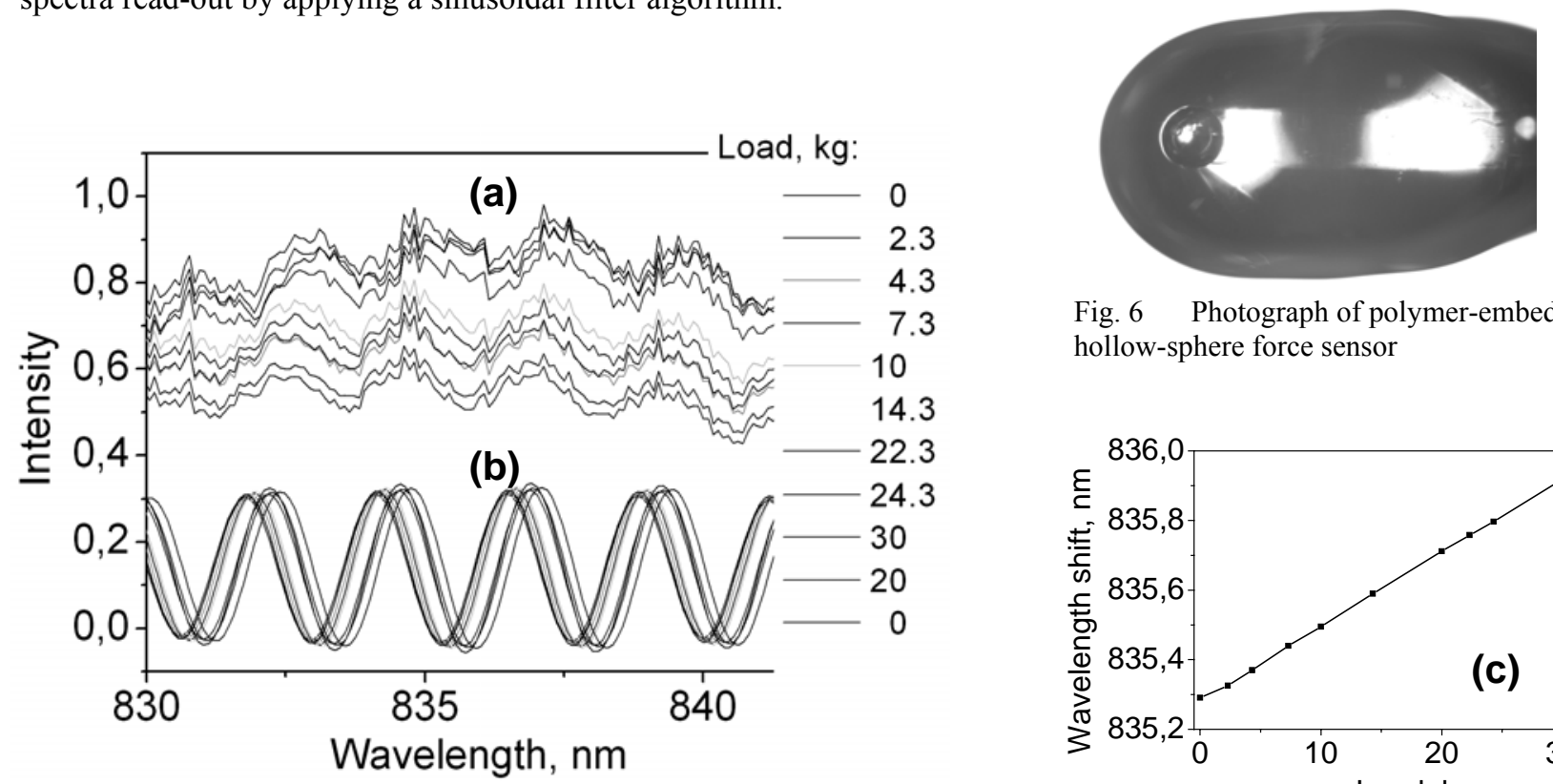

Fig. 6 Photograph of polymer-embedded hollow-sphere force sensor

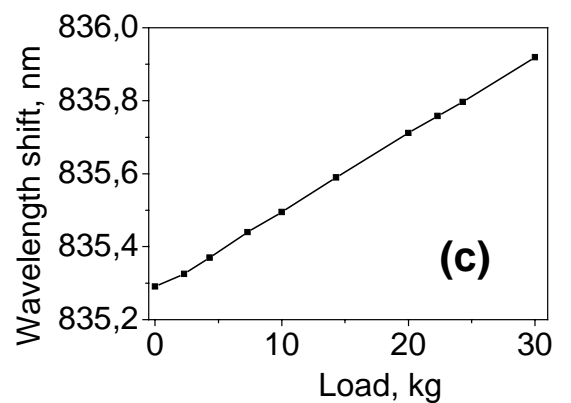

Fig. 7 Reflective spectra of embedded hollow sphere force sensor; spectral shift due to applied tensile force, of magnitude shown in the diagram

a (left top): unfiltered spectra; b (bottom): via sinusoidal filter, bandpass $0.4 .0 .45 \mathrm{~nm}^{-1} ; \quad \mathrm{c}$ (right): wavelength shift (b) vs. load

\section{CONCLUSIONS}

We have shown improved sensitivity for a hollow glass sphere pressure sensing method, when addressed by a polychromator-based grating interrogation system, and have, for the $1^{\text {st }}$ time, shown how this simple sensor can also be used to measure strain, when embedded in a simple glass-fibre-reinforced load-cell element. Unlike many other micromechanical pressure sensing structures, the sensor head is intrinsically sealed. Processing the spectrum with a sinusoidal filter of appropriate spacing improves signal to noise. We have also conducted initial dynamic measurements with the sensor, and hope to report more details of these measurements, firstly at the meeting and in future publications.

\section{ACKNOWLEDGEMENTS}

The authors like to thank PQ Potters Europe GmbH, Germany, for providing free samples of their hollow glass spheres, and University of Southampton for allowing a period of study leave for J.P. Dakin.

\section{REFERENCES}

[1] Xu M.G., Dakin J.P. "A novel hollow-glass microsphere sensor for monitoring high hydrostatic pressure", Proceedings of SPIE Vol. 1795 "O/E fibers '92 - Fiber optic \& laser sensors", pp. 2-7 (1992)

[2] Willsch R., Ecke W., Bartelt H. "Optical Fiber Grating Sensor Networks and their Application in Electric Power Facilities, Aerospace and Geotechnical Engineering", Proc. of 15th International Conf. on Optical Fiber Sensors, Portland/Oregon, Technical Digest, pp. 49-54, IEEE, Piscateway, NJ (2002)

[3] Roark R.J., Young W.C. "Formulas for stress and strain", Mc Graw Hill, 6th edition, p. 451 (1989)

[4] Ecke W., Latka I., Willsch R., Reutlinger A., Graue R. "Fibre Optic Sensor Network for Spacecraft Health Monitoring", Measurement Science Technology, Vol. 12, pp. 974-980 (2001) 